AperTO - Archivio Istituzionale Open Access dell'Università di Torino

\title{
Use of Biowaste-Derived Biosurfactants in Production of Emulsions for Industrial Use
}

\section{This is the author's manuscript}

Original Citation:

\section{Availability:}

This version is available http://hdl.handle.net/2318/145310

since 2016-01-08T10:45:01Z

Published version:

DOI:10.1021/ie4037609

Terms of use:

Open Access

Anyone can freely access the full text of works made available as "Open Access". Works made available under a Creative Commons license can be used according to the terms and conditions of said license. Use of all other works requires consent of the right holder (author or publisher) if not exempted from copyright protection by the applicable law. 


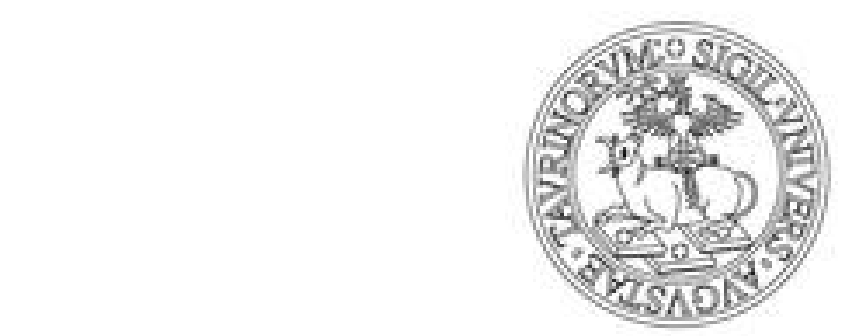

\section{UNIVERSITÀ DEGLI STUDI DI TORINO}

This is an author version of the contribution published on:

Adriana K. N. Vargas,Alessandra Bianco Prevot,Enzo Montoneri,Galo

Carrillo Le Roux,Piero Savarino,Roberta Cavalli,Roberto Guardani,Silvia Tabasso

Use of Biowaste-Derived Biosurfactants in Production of Emulsions for Industrial Use

INDUSTRIAL \& ENGINEERING CHEMISTRY RESEARCH (2014) 53

DOI: $10.1021 / \mathrm{ie} 4037609$

The definitive version is available at:

http://pubs.acs.org/doi/abs/10.1021/ie4037609 


\title{
Use of Biowaste-Derived Biosurfactants in the
}

\section{Production of Emulsions for Industrial Use}

\author{
Adriana $K$ N Vargas, ${ }^{* a, b}$ Piero Savarino, ${ }^{a}$ Enzo Montoneri, ${ }^{c}$ Silvia Tabasso, ${ }^{a}$ Roberta Cavalli, ${ }^{d}$ \\ Alessandra Bianco Prevot, ${ }^{a}$ Roberto Guardani, ${ }^{b}$ Galo A.C Roux ${ }^{b}$
}

${ }^{a}$ Universita` di Torino, Dipartimento di Chimica, Via P. Giuria 7, 10125 Torino, Italy

${ }^{b}$ University of São Paulo, Chemical Engineering Department, Av. Luciano Gualberto 380 tv 3, 05508-900 São Paulo SP, Brazil

${ }^{\mathrm{c}}$ University of Foggia, STAR*Integrated Research Unit, Via Gramsci, 89-91, 71121 Foggia, Italy

${ }^{\text {d} U n i v e r s i t a ` ~ d i ~ T o r i n o, ~ D i p a r t i m e n t o ~ d i ~ S c i e n z a ~ e ~ T e c n o l o g i a ~ d e l ~ F a r m a c o, ~ V i a ~ G i u r i a ~ 9, ~} 10125$ Torino, Italy

\section{Corresponding author}

*Email address: akninov@usp.br Tel.:+551130912237; fax: +551130912246

KEYWORDS: emulsion, surfactant, metalworking fluid, metalworking process, biobased substances, waste management 
ABSTRACT: This study reports a study on the use of soluble bio-based substances (SBO) with emulsifying properties in the production of oil-in-water emulsions for industrial consumption. One of the potentially interesting applications is in the formulation of metalworking fluids, which typically consist of oil-in-water emulsions used in metal manufacturing industries. Soluble bio-based substances (SBO) were collected from urban food and gardening wastes in a waste management plant located in a densely populated area in North Italy. The biowastes were fermented under anaerobic and/or aerobic conditions, and then treated chemically to yield four different SBO samples, which were characterized for chemical composition, solution behavior by water surface tension measurements, and ability to form stable $10 \%$ oil-in-water $(\mathrm{O} / \mathrm{W})$ emulsions. Experiments were carried out to evaluate the hydrophilic-lipophilic balance of emulsions with surfactant concentrations in the $2-8 \mathrm{w} / \mathrm{w} \%$ range. Stable emulsions were obtained with at least $3 \mathrm{w} / \mathrm{w} \%$ surfactant concentrations. All SBO samples presented the characteristics necessary for acting as emulsifiers, when compared with conventional emulsifiers. According to rheology, particle size and centrifugation tests, SBO samples isolated from composted biowastes performed better than samples isolated from the digestate of anaerobicallyfermented biowastes. The product ash content and/or isolation process also affect the emulsifier performance. The effects of the biowaste source, chemical composition, and surface activity on the emulsifier performance were evaluated, indicating the feasibility of attaining stable emulsions with environmental benefits. 


\section{INTRODUCTION}

Soluble bio-based substances (SBO) are compounds isolated from compost of green residues produced by aerobic and anaerobic process, which have surfactant properties and thus potential technological applications, as presented by Montoneri et al., ${ }^{1}$ Savarino et al. ${ }^{2}$ and Bianco Prevot et al. ${ }^{3}$ The advantage with respect to commercial surfactants is that SBO do not cause negative environmental impacts due to their compatibility with natural soil and organic matter. ${ }^{4}$ This fact is confirmed by agriculture, ${ }^{5,6}$ and animal $^{7}$ studies demonstrating that SBO can promote safe plant growth and farming practices.

SBO are described as a mix of macromolecules with average molecular weight ranging from 67 to $463 \mathrm{~kg} \mathrm{~mole}^{-1}$ and containing several functional groups and organic functions of different polarity as represented in the molecular fragment shown in Figure 1. The organic moieties shown in Figure 1 are remaining parts of the main constituents of the sourcing biowaste, which are not completely mineralized during aerobic and/or anaerobic biodegradation. The molecular fragments shown in Figure 1 are responsible for the observed behavior of SBO as surfactants and/or polyelectrolytes, with a wide range of configurations due to the relative ratios of the different organic functions. ${ }^{8}$

One of the potential applications of SBO is as surfactants in the formulation of emulsions for industrial use, such as metalworking fluids (MWF). These oil-in-water $(\mathrm{O} / \mathrm{W})$ emulsions have their behavior largely dependent on the formulation. According to Wilbert, ${ }^{9}$ MWF with 5-50 \% of oil dispersed in water are designed to act as lubricants and cooling fluids, and to remove metallic particles produced in metalworking processes. Stability and viscosity are thus essential characteristics of such emulsions. During the machining processes, accumulation of contaminants such as extraneous oil, and bacteria can affect negatively the MWF functionality. 
The disposal of aged MWF in the environment can produce significant impact. For this reason, the development of new technologies for minimizing the environmental impact caused by effluents from metal working processes is an ongoing effort. Recently published studies show alternatives for emulsion treatment. Some authors ${ }^{10}$ propose the treatment of aged emulsions by coagulation, centrifugation, ultrafiltration, and vacuum evaporation. Others ${ }^{11}$ have studied mixed anionic/nonionic emulsifier systems for petroleum and bio-based MWF to improve emulsion stability and lifetime under hard water conditions, a common cause of emulsion destabilization leading to MWF disposal. Greeley and Rajagopalan ${ }^{12}$ analyzed the impact of natural contaminants on the lubricating, cooling, corrosion inhibition, and surface finishing functionalities of MWFs in order to minimize their destabilization and optimize their management.

Another alternative consists in substituting one or more emulsion components with ecofriendly compounds. Zhang and coworkers, ${ }^{13}$ for instance, carried out experiments to replace conventional oil with castor oil by studying the stability of emulsions with different formulations. The demand for MWF can be an attractive factor for developing ecofriendly components such as SBO. As an example, in 2006 the U.S. market consumed over 20 million liters of MWF and 600 million liters of automotive and industrial lubricants. ${ }^{14}$ In terms of economic feasibility, a preliminary estimation can be made as follows. By assuming a price of $4.5 €$ per liter of MWF, and SBO production cost between 0.1 and $0.5 € / \mathrm{kg},{ }^{4}$ with capital cost for a plant producing $500 \mathrm{SBO}$ tons per year of about $500.000 €$, the SBO products can be sold in the chemical market for ca. $1 € / \mathrm{kg}$. This represents a net revenue of ca. 250 thousand euros per year. Considering an emulsion containing $2.5 \% \mathrm{SBO}$, the cost contribution of this additive in the 
finished emulsified product would be less than $0.02 € / \mathrm{L}$. Thus, the $1 € / \mathrm{kg}$ purchase price can be acceptable by potential users.

The present study evaluates the use of SBO as surfactants in MWF based on the stability and viscosity of $\mathrm{O} / \mathrm{W}$ emulsions using different $\mathrm{SBO}$ samples obtained from different sources, as alternatives to synthetic surfactants. Thus, four SBO samples, characterized by different origin, chemical processing, chemical composition and surface tension properties were tested for their potential to yield ecofriendly stable emulsion formulations to be used as MWF in metalworking processes.

\section{EXPERIMENTAL}

\subsection{Materials}

SBO are hydrolysates with molecular cut-off above 5000 D. They contain water-soluble polymeric molecules, which may range up to several hundred $\mathrm{kg} \mathrm{mole}^{-1} .{ }^{8}$ Due to their complexity, SBO cannot be analyzed as well as synthetic molecules. Their chemical nature can at best be identified by the functional groups concentration, as reported in Table 1. These organic moieties are the likely memory of the main constituents of the sourcing bioorganic refuse matter, which are not completely mineralized during aging under fermentation conditions. For this reason, SBO may be considered the pristine material of natural soil organic matter formed under longer aging conditions. Thus, the structural similarities between SBO and natural soil organic matter are not surprising.

The other reagents used to prepare the emulsions were sodium dodecyl sulfate (SDS) , BRIJ72, and paraffin oil 100\%, all purchased from Aldrich and used as received, and deionized water. 


\subsection{Methods}

The SBO samples were produced from municipal biowastes sampled from three different streams of the ACEA Pinerolese waste treatment plant in Pinerolo (Italy). The sources are: a) the digestate (FORSUD) recovered from a biogas production reactor fed with the organic humid fraction from separate source collection of urban refuse; b) the compost (CV) obtained from urban private garden and public park trimming residues aged for 230 days; and c) the compost (CVDF) obtained from a 35/55/10 w/w/w FORSUD/home gardening and park trimming residues/sewage sludge (SS) mix, collected after 110 days composting under aerobic conditions. These materials were processed in a pilot plant made available from Studio Chiono e Associati in Rivarolo Canavese, Italy. This comprised an electrically heated mechanically stirred $500 \mathrm{~L}$ reactor, a $102 \mathrm{~cm}$ long x $10.1 \mathrm{~cm}$ diameter polysulfone ultrafiltration membrane with $5 \mathrm{kD}$ molecular weight cut-off supplied by Idea Engineering s.r.l. from Lessona (Bi), Italy, and a forced ventilation drying oven. According to the operating experimental conditions, the biowaste was reacted during $4 \mathrm{~h}$ with $\mathrm{pH} 13$ water at $60{ }^{\circ} \mathrm{C}$ and $4 \mathrm{~V} / \mathrm{w}$ water/solid ratio. The liquid/solid mix was allowed to settle to separate the supernatant liquid phase containing the soluble hydrolysate from the insoluble residue. The recovered hydrolysate was circulated at $40 \mathrm{~L} \mathrm{~h}^{-1}$ flow rate through the ultrafiltration membrane operating with tangential flow at 7 bar inlet and 4.5 bar outlet pressure to yield a retentate with $5-10 \%$ dry matter content. The retentate was finally dried at $60{ }^{\circ} \mathrm{C}$ to yield the solid SBO samples in $15-30 \% \mathrm{w} / \mathrm{w}$ yield, relatively to the starting biowaste dry matter. The SBO samples are hereinafter referred to with the acronym of the sourcing biowaste. These products were found to contain about 15-28 \% ash. The ash consisted of the added alkali cation of the hydrolyzing base, as well as other mineral components present in the sourcing biowaste, containing $\mathrm{Si}, \mathrm{Ca}, \mathrm{Mg}, \mathrm{Al}$ and $\mathrm{Fe}$. Ash content was determined by 
calcining each sample at $650{ }^{\circ} \mathrm{C}$ for 4 hours, according to the procedure described in Matthiessen et al. ${ }^{15}$ An aliquot of the CV SBO was further washed with $\mathrm{HCl}$, water, $\mathrm{HF}$, again with water, and then dried at $60{ }^{\circ} \mathrm{C}$ to yield the low ash CV SBO (CVla) product. This sample contained $99.1 \%$ volatile solids as determined via calcination at $650{ }^{\circ} \mathrm{C}$.

The SBO emulsifying capacity was tested for the preparation of $\mathrm{O} / \mathrm{W}$ emulsions containing 10 $\%$ paraffin oil in water, as a typical composition for machining or grinding fluids. ${ }^{14}$ The nonionic (NI) and anionic (AN) surfactants were dissolved in previously heated oil at approx. $80^{\circ} \mathrm{C}$, and in $\mathrm{pH} 8$ water at room temperature. The water solution was slowly added to the oil solution and mixed for 5 minutes by means of a magnetic stirrer, forming a pre-emulsion. This was then mixed for 5 additional minutes using a stator-rotor device at $11000 \mathrm{rpm}$. $\mathrm{O} / \mathrm{W}$ mixtures were made at $2-8 \mathrm{w} / \mathrm{w} \%$ total surfactant concentration and different AN/NI surfactant ratios (R). The relative surfactant composition in the samples was calculated according to the literature, ${ }^{13}$ based on eqs 1 to 3 :

$$
\begin{array}{r}
H L B_{\text {required }}=\sum_{i=1}^{n} X_{i} H L B_{i} \\
X_{A N}=\frac{H L B_{\text {required }}-H L B_{N I}}{H L B_{A N}-H L B_{N I}} \\
X_{N I}=\left(1-X_{A N}\right)
\end{array}
$$

Where $X_{A N}$ and $X_{N I}$ are the mass fractions of the AN and NI surfactants, $n$ is the number of surfactant species, and $H L B$ is the hydrophilic-lipophilic balance parameter for the oil to be emulsified $\left(H L B_{\text {required }}\right)$, for the AN $\left(H L B_{A N}\right)$, and for the NI $\left(H L B_{I N}\right)$ surfactants. According to Griffin, ${ }^{15}$ the HLB is calculated based on the hydrophilic and lipophilic portions of a single molecule or of a mixture of molecules (eq 1). For the specific case of this work, only the HLB values for paraffin oil and the non-ionic BRIJ72 surfactant were available, ${ }^{16}$ i.e., $\mathrm{HLB}_{\text {paraffin oil }}=$ 
10 and $\mathrm{HLB}_{\mathrm{BRIJ72}}=4.9$. For the anionic SBO surfactant the HLB could not be estimated. These substances are a complex mixture of molecules of different chemical nature and molecular weight. Aside from the types and relative ratios of the functional groups in Table 1, the chemical nature and relative ratios of the single components of the SBO molecular pool are not known. Under these circumstances, the $\mathrm{O} / \mathrm{W}$ emulsions used in this study were prepared by gradually adding each component and observing the result, as suggested by ICI Americas Inc. ${ }^{17}$ A number of surfactant mixtures were prepared with different $X_{A N}$ and $X_{N I}$ values, and the HLB values of the SBO samples $\left(H L B_{A N}\right)$ were estimated by means of eq 2, by using the $\mathrm{X}_{\mathrm{AN}}$ value for which the most stable emulsion was obtained. Emulsion stability was determined by centrifuging the total volume of the oil and water sample (Vt) for $10 \mathrm{~min}$ under $4000 \mathrm{rpm}$, followed by measuring the separated non-emulsified volume (Vne), and calculating the parameter $F$, where $F=V n e / V t$. The experiments were performed in a multispeed refrigerated centrifuge ALC PK $131 \mathrm{R}$ at $20^{\circ} \mathrm{C}$. All samples were prepared in triplicates.

Emulsion samples with $5 \mathrm{wt} \%$ surfactant were diluted 1:1200 in deionized water, in order to enable the measurement of the droplet size distribution. These conditions have been defined in previous studies by the authors, ${ }^{18,19}$ in which it was verified that the dilution did not affect the droplet size distribution. The measurements were carried out using a Coulter Counter instrument (Multisizer IIe, Coulter International Corporation). The Sauter mean diameter, $d_{3,2}$, was used to characterize the droplet size distribution of the samples (eq 4).

$$
d_{3,2}=\frac{\sum n_{i} d_{i}^{3}}{\sum n_{i} d_{i}^{2}}
$$

where $n$ is the number of droplets with equivalent spherical diameter $d$ in droplet class $i$.

Rheology measurements were made in a Brookfield DV-III Ultra rheometer. The experiments were carried out at constant temperature and pressure, and at $20 \mathrm{rpm}$ spindle velocity. 


\section{RESULTS AND DISCUSSION}

\subsection{SBO characterization}

The results of the characterization of the SBO samples are summarized in Table 1. The functional groups reported are probably not homogeneously distributed over the entire molecular pool. Under these circumstances, in order to investigate the relationship between the chemical nature and properties of the products and their performance, the SBO have been ranked according to the empirical parameters LH and Af/Ar defined in Table 1. LH provides an indication of the degree of lipophilicity and Af/Ar is an index of the type of liphophilicity. FORSUD is the most lipophilic SBO, followed in the order of decreasing lipophilicity by CVDF and CV. The CVla SBO appears to have lower Af/Ar index and higher LH values than CV.

\subsection{Surface tension of SBO}

For the purpose of this work, the SBO samples were characterized for their surface activity properties in aqueous solution yielding plots of surface tension $(\gamma)$ versus SBO concentration (Cs) (Figure 2). As previously reported by Montoneri and co-workers, ${ }^{8}$ these measurements provide hints on the molecular conformation of the investigated substances in solution. The SBO samples yielded $\gamma$-Cs plots apparently similar to those of small molecule surfactants, i.e., showing a breaking point with slope $\left(\mathrm{d} \gamma_{\mathrm{i}} / \mathrm{dCs}\right)$ change. According to Montoneri and co-workers, ${ }^{8}$ with small molecule surfactants such as sodium dodecyl sulfate, SDS, it is easy to visualize conformational changes from the slope of the $\gamma$-Cs plots. In essence, at low Cs single molecules occupying the air-water interface may be idealized lying orthogonal to the water surface, dipping their hydrophilic heads in the water phase and pointing their hydrophobic tails toward the air 
phase. In the bulk water phase, molecular interactions between single molecules are expected to yield more or less spherical or cylindrical micelles where the polar heads of each molecule are oriented towards the external water phase and the hydrophobic segments form the inner lipophilic core. With complex polymeric molecules, as represented in Figure 1, one could expect that the single molecules at the air-water interface acquire a more or less flat configuration to point their hydrophilic heads toward the water phase. In the bulk water phase, two conformations are possible, that is, single molecules arranged in coil form to yield pseudomicelles with the external hydrophilic surface pointing toward the water phase and the inner lipophilic molecular segments away from it, or aggregates of single molecules to yield large micelles. The formation of pseudomicelles does not necessarily exclude the formation of molecular aggregates. Intermolecular ionic or H-bonding interactions between pseudomicelles are likely to occur and become more important at higher concentration. The decrease in the concentration of water molecules in SBO solution implies shortening the intermolecular H-bonding network and a decrease in the distance between pseudomicelles, eventually leading to polymeric micelles. Formation of such large aggregates is likely to occur at 50-100 $\mathrm{g} \mathrm{L}^{-1} \mathrm{Cs}$. Under these conditions, the product is no longer soluble in water and a viscous gel-like phase separates from the bulk water phase. Thus, whereas the $\gamma$-Cs plot for the SBO may indicate that these substances perform apparently like small molecule surfactants at $\mathrm{Cs}<10 \mathrm{~g} \mathrm{~L}^{-1}$, at $\mathrm{Cs}>50 \mathrm{~g} \mathrm{~L}^{-1}$ they behave more like water-soluble polyelectrolytes.

The surface tension measurements evidenced another important behavior of SBO in solution. The surface tension of the solutions at $\mathrm{Cs}<10 \mathrm{~g} \mathrm{~L}^{-1}$ was found to decrease continuously with increasing time from the preparation. The rate of decrease was high at the beginning and tended to lower values for longer times. However, a stable value was not reached even after $48 \mathrm{~h}$. Due to 
this behavior, all values were recorded 200 minutes after solution preparation, when the surface tension decrease was rather low. Although the measurements at this time did not provide an absolutely stable surface tension value, the relative differences between the investigated SBO were constant. The observed difficulty of SBO to reach a stable conformation in solution is probably due to difficulties of reorientation of the high molecular weight surfactants at the interface. $^{20}$

In order to clarify the differences of surface tension properties among the SBO samples investigated in this study, each $\gamma$-Cs plot was divided into two segments, which were analyzed by fitting a straight line by least squares (Figure 2). Table 2 shows the slope values obtained for each plot $\left(\mathrm{d} \gamma_{\mathrm{i}} / \mathrm{dCs}, \mathrm{mN} \mathrm{L} \mathrm{m}^{-1} \mathrm{~g}^{-1}, \mathrm{i}=1,2\right)$ and the associated coefficient of determination $\left(\mathrm{R}_{\mathrm{i}}, \mathrm{i}=\right.$ $1,2)$, together with the concentration $\left(\mathrm{Cs}_{1}\right)$ at which the slope change occurs and the corresponding surface tension value $\left(\gamma \mathrm{Cs}_{1}\right)$. The high $\mathrm{R}_{\mathrm{i}}$ values $(\geq 0.90)$ observed in most cases indicate that the linear fitting resulted in an adequate mathematical representation of the experimental $\gamma$-Cs plots. The data enable a quantitative evaluation of the differences among the four investigated SBO samples. In essence, the FORSUD SBO resulted in the largest $\mathrm{d} \gamma_{1} / \mathrm{dCs}$, the lowest $\mathrm{Cs}_{1}$ and the second $\gamma \mathrm{Cs}_{1}$ values, whereas no significant difference was observed between CV and CVDF samples. The values of $d \gamma_{1} / \mathrm{dCs}$ and $\mathrm{Cs}_{1}$ are larger for CVla compared to $\mathrm{CV}$, whereas a lower value of $\gamma \mathrm{Cs}_{1}$ was obtained for CV1a than for $\mathrm{CV}$.

\subsection{HLB analysis}

Preliminary tests were carried out for estimating the HLB of each SBO sample. Thus, mixtures of the commercial BRIJ72 non ionic (NI) surfactant and one at a time of the four different anionic (AN) SBO surfactants were prepared. Emulsions with $2-8 \mathrm{w} / \mathrm{w} \%$ total surfactant 
concentration and AN/NI surfactants ratio $(\mathrm{R})$ in the range from 0.47 to 1.0 were prepared to yield the estimated HLB values shown in Table 3. These samples were left to stand for several days and the time to visually observe droplets or deposit was recorded. The results in Table 3 show that emulsions containing $8 \%$ total surfactant concentration were stable over the whole range of $\mathrm{R}$ values, except the sample containing $\mathrm{CVDF}$ at $\mathrm{R}$ equal to 0.72 . At lower total surfactant concentration, the number of unstable emulsion samples increased. At $2 \%$ total surfactant concentration, no sample was stable. The samples containing CVDF were apparently the most stable ones. These samples were stable over the whole monitoring time, i.e., 15 days, when the total surfactant concentration remained above $3 \%$, and for $\mathrm{R}$ values ranging from 0.51 to 0.61 . It is interesting to observe that, at $3 \%$ total surfactant concentration, the sample containing CVla yields a stable emulsion for $\mathrm{R}=0.51$, whereas the sample prepared with $\mathrm{CV}$ yielded no stable emulsion within the range of $\mathrm{R}$ values studied. At total surfactant concentration of $5 \%$ or higher all samples resulted in stable emulsions. The results indicate that $3 \%$ total surfactant concentration might be a limiting value to enable the discrimination among the investigated SBO samples in terms of their HLB. Based on the emulsions that were stable for longer periods of time at $3 \%$ total surfactant concentration, the HLB values for each SBO were estimated as 17.5 for FORSUD, 18 for CV, 20 for CVla, and 18 to 20 for CVDF.

\subsection{Droplet size distribution in emulsions with SBO}

The decrease of emulsion stability when the total surfactant concentration decreases is a known effect, related to an increase in the droplet coalescence process, eventually leading to emulsions showing multimodal droplet size distributions, as reported in the literature. ${ }^{18,19}$ Hence, a population of large droplets is formed in the emulsion when the amount of surfactant is 
not enough to maintain the emulsion stable for long periods of time. Figure 3 illustrates this effect visually for emulsions made with $\mathrm{CV}$ as surfactant at two concentrations, and $\mathrm{R}$ equal to 0.61. Figure 4 shows the droplet size distribution for emulsions containing 3 and $5 \%$ total surfactant concentration, and each $\mathrm{SBO}$ at the $\mathrm{R}$ value that yielded the most stable emulsions (see Table 3), measured 8 days after preparation. At $3 \%$ total surfactant concentration, stable emulsions made with CVDF and CVla showed droplet sizes ranging from 1.56 to $2.19 \mu \mathrm{m}$ and Sauter mean diameter equal to $1.86 \mu \mathrm{m}$. The less stable emulsions showed droplets in the same size range, as well as larger droplets, too. For CV the droplet size ranged from 1.56 to $2.18 \mu \mathrm{m}$, and from 6.57 to $8.34 \mu \mathrm{m}$. For FORSUD the droplet size ranged from 1.87 to $2.19 \mu \mathrm{m}$, and from 5.21 to $7.71 \mu \mathrm{m}$. The Sauter mean diameter values for CV and FORSUD were respectively 2.63 and $2.78 \mu \mathrm{m}$, which is significantly higher than those for CVDF and CVla. At $5 \%$ total surfactant concentration all emulsions were stable. As shown in Figure 4, these samples contained droplets that are smaller than $3.54 \mu \mathrm{m}$ and Sauter mean diameter less than $2.60 \mu \mathrm{m}$. This is an effect of the coalescence inhibition caused by the increased surfactant concentration.

\subsection{Centrifugation tests}

Further tests were carried out in order to evaluate emulsion stability based on the values of the parameter $\mathrm{F}$ after centrifugation (see Experimental section). In this case, emulsions containing SBO were compared with emulsions containing the commercial sodium dodecyl sulphate (SDS) surfactant with HLB equal to 40 , based on procedures in the literature. ${ }^{21}$ The $\mathrm{R}$ value for the SDS emulsion was 0.17. Based on the HLB values and on Eqs. 1 to 3, this surfactant/co-surfactant composition is supposed to yield the most stable emulsions. However, Eqs. 1 to 3 are based on the assumption that the HLB is a sufficient stability criterion while, in fact, stability also depends 
on the chemical functions that are present in the surfactants. This means that mixtures of different surfactant types may yield the same required HLB, but exhibit different efficiencies in terms of emulsion stability. ${ }^{16}$ Figure 5 illustrates this situation by means of the parameter F. For all tested values of total surfactant concentration, the emulsion prepared with SDS was less stable when processed by centrifugation than the emulsions prepared with SBO. The performances of emulsions prepared with the four SBO samples at total surfactant concentration less than $4 \mathrm{w} / \mathrm{w} \%$ are similar. However, for higher concentrations, different performances were observed. The FORSUD sample resulted in intense phase separation at $2 \mathrm{w} / \mathrm{w} \%$ total surfactant concentration, while at $5 \mathrm{w} / \mathrm{w} \%$ total surfactant concentration it performed significantly better. Samples CVDF and CVla were apparently the best stabilizers at $5 \mathrm{w} / \mathrm{w} \%$ total surfactant concentration. Particularly for emulsions prepared with CVDF no phase separation was observed. The comparison of CV and CVla data indicates that the SBO emulsifier performance was possibly affected by the presence of mineral compounds, since the presence of inorganic salts contribute to destabilize emulsions. ${ }^{22}$ The higher viscosity is the reason for the higher centrifugation stability of the SBO emulsions compared to the SDS emulsions. However, in order to make a comparison between the biosurfactants and the commercial synthetic surfactants the viscosity of the two emulsions should be similar. Based on the performance of each emulsion sample (Table 3 and Figures 3 to 5) and on the chemical and surface tension data of each SBO sample, no correlation is apparent between product performance and surface activity. The CVDF and CVla SBO samples, which have 5.3-4.5 LH and 1.3-0.8 Af/Ar values, showed the largest HLB values, i.e., 18-20, and yielded the most stable emulsions, based on the number of days of emulsion stability shown in Table 3, and the F values in Figure 5. The other two SBO samples are predominantly aliphatic, as indicated by the Af/Ar index, which is 3.3 for FORSUD and 1.8 
for CV. Compared to CVDF and CVla, FORSUD is more lipophilic (LH equal to 9.3) and CV is more hydrophilic (LH equal to 3.6). Therefore the performance of SBO as emulsifiers is strongly dependent on their chemical composition and on their biowaste sources and previous treatment. There is apparently an optimal composition and hydrophilicity range associated with the best performance, whereas SBO samples with hydrophilicity values outside the optimal range (i.e. with LH values higher or lower than 5.3-4.5) exhibit lower performance. ${ }^{23-25}$

\subsection{Rheological properties}

For the practical use of surfactants as emulsifiers in emulsions of industrial interest, such as metalworking fluid formulation, for example, the emulsion stability is certainly desirable, but is not the only requirement. The emulsion rheological properties are also important. In order to evaluate the potential of SBO to yield emulsion formulations with adequate performance under real metalworking process conditions, rheology measurements were also carried out. Figure 6 shows shear stress versus shear rate results for the emulsions made with the four SBO surfactants at $5 \% \mathrm{w} \%$ total surfactant concentration and $\mathrm{R}$ values for which the most stable emulsions were obtained, i.e., 0.61 for CV, 0.51 for CVla, 0.57 for CVDF, and 0.67 for FORSUD (see Table 3). The stress response of all SBO emulsions is not linear and the curve starts from the origin. This is characteristic of pseudoplastic fluid behavior. Figure 7 shows the change of viscosity with time after preparation for the same samples. All emulsions exhibited decreasing viscosity over time, in the first two days after emulsion preparation. The decrease of viscosity with time is more pronounced for SBO than for the SDS-based emulsions. This may reflect a slower process of stabilization in solution for the SBO samples, as was also evidenced by the surface tension measurements. Samples prepared with CVDF yielded the most viscous emulsions, followed by 
CVla, FORSUD and CV. The high viscosity for CVDF and CVla has probably contributed to the higher stability observed in the centrifugation tests (Figure 5). Indeed, at low viscosity the possibility that emulsion droplets collide and coalesce during Brownian motion increases. ${ }^{14}$ Viscosity is also critical to determine the quality of the lubricant film. In metal working applications, it determines the effectiveness of the film in separating the tool from the work piece and therefore controlling friction and wear. However, fluids with higher viscosity are less efficient in removing chips and in cooling. Hence optimum viscosity must be estimated for each operation. ${ }^{26}$ According to the results in Figure 7, all viscosity values of the SBO emulsions are above $1000 \mathrm{cP}$, which is more than double the typical viscosity of commercial emulsions based on SDS. The high viscosity of the SBO emulsions is possibly a consequence of the polymeric nature of the molecules composing these substances and their capacity to yield large aggregates between macromolecules. The interactions between aggregates are expected to yield a network of inter-aggregates and H-bonds which can favor the formation of viscous gel-like substances. This behavior distinguishes the polymeric SBO surfactants from small molecule surfactants. Thus, the use of SBO in emulsions for which viscosity control is necessary involves blending with surfactants composed of small molecules.

\section{CONCLUSIONS}

The present comparative evaluation of the behavior of biowaste-derived biosurfactants (SBO) from different sources considered their use as surfactants in $\mathrm{O} / \mathrm{W}$ emulsions of industrial interest. Metalworking fluids were considered as a potential application, and the evaluation was based on stability and rheological behavior of the emulsions. 
The results indicate that the performance of the SBO is strongly dependent on its constitution, which depends on the biowaste source. All the SBO samples resulted in emulsions that were more stable than the one obtained with a conventional surfactant (sodium dodecyl sulphate). The experiments have indicated an apparently optimal composition and hydrophilicity range associated with the stability of the emulsions, whereas SBO samples with hydrophilicity values outside an optimal range, which corresponds to $\mathrm{LH}$ values from 5.3 to 4.5 , exhibit lower performance.

The viscosity of the SBO-based emulsions were above $1000 \mathrm{cP}$ for all samples, which is more than double the typical viscosity of commercial emulsions based on sodium dodecyl sulphate as surfactant. The high viscosity of the SBO emulsions is possibly a consequence of the nature of the molecules composing these substances and their capacity to yield large aggregates between macromolecules. Two of the SBO samples, with a definite composition and hydrophilicity range, were identified as best emulsion stabilizers. The viscosity can, however, be to high with respect to requirements for use in some applications. In these cases, mixtures of SBO and small molecule anionic surfactants may enable the development of formulations with tailored viscosity.

These results are a representative figure of the behavior of SBO biosurfactants, which can contribute to the development of an attractive component for the production of technically and economically feasible ecofriendly $\mathrm{O} / \mathrm{W}$ emulsions as lubricants.

\section{- ACKNOWLEDGEMENTS}

This work was carried out with funds from the Ministero delle Politiche Agricole e Forestali for the Agrienergia project, Brazilian federal agency CNPq, BRAGECRIM Program, and the mobility project IRSES Marie Curie 2011. The authors are also thankful to the following private and/or public institutions: (a) Acea Pinerolese Spa in Pinerolo (TO) for supplying the UR 
extracts sourcing18biowaste; (b) Studio Chiono ed Associati in Rivarolo Canavese (TO) for making available pilot equipment and services for the production of the Biowaste extracts.

\section{- REFERENCES}

(1) Montoneri, E.; Boffa, V.; Savarino, P.; Tambone, F.; Adani, F.; Micheletti, L.; Gianotti, C.; Chiono, R. Use of Biosurfactants from Urban Wastes Compost in Textile Dyeing and Soil Remediation. Waste Manag. 2009, 29, 383.

(2) Savarino, P.; Montoneri, E.; Bottigliengo, S.; Boffa, V.; Guizzetti, T.; Perrone, D.G.; Mendichi, R. Biosurfactants from Urban Wastes as Auxiliaries for Textile Dyeing. Ind. Eng. Chem. Res. 2009, 48, 3738.

(3) Bianco Prevot, A.; Avetta, P.; Fabbri, D.; Laurenti, E.; Marchis, T.; Perrone D. G.; Montoneri, E.; Boffa, V. Waste-Derived Bioorganic Substances for Light-Induced Generation of Reactive Oxygenated Species. Chem. Sus. Chem. 2011, 4, 85.

(4) Montoneri, E.; Mainero, D.; Boffa, V.; Perrone, D. G; Montoneri, C. Biochemenergy : A Project to turn an Urban Wastes Treatment Plant into Biorefinery for the Production of Energy, Chemicals and Consumer's Products with Friendly Environmental Impact. IJGEnvI. 2011, 11, 170.

(5) Negre, M.; Mozzetti Monterumici, C.; Vindrola, D.; Piccone, G.; Perrone, D. G.; Tomasso, L.; Montoneri, E. Monterumici Horticultural and Floricultural Applications of Urban Wastes Originated Fertilizers. Compost. Sci. Util. 2012, 20, 150. 
(6) Sortino, O.; Dipasquale, M.; Montoneri, E.; Tomasso, L.; Avetta, P.; Bianco Prevot, A. 90\% Yield Increase of Red Pepper with Unexpectedly Low Doses of Compost Soluble Substances. Agron. Sustainable Dev. 2013, 33, 433.

(7) Dinuccio, E.; Biagini, D.; Rosato, R.; Balsari, P.; Lazzaroni, C.; Montoneri, E. Use of Acid Soluble Bio-organic Substances Extract as Rabbits Feed Additive to Reduce Manure Gases Emission During Storage. Presented at the Advances in Animal Biosciences: Proceedings of the 5th Greenhouse Gases and Animal Agriculture Conference, Dublin, Ireland, June, 2013; p 515.

(8) Montoneri, E.; Boffa, V.; Savarino, P.; Perrone, D. G.; Montoneri, C.; Mendichi, R.; Acosta, E. J.; Kiran, S. Behavior and Properties in Aqueous Solution of Biopolymers Isolated from Urban Refuse. Biomacromolecules. 2010, 11, 3036.

(9) Wilbert, J. O. Lubricants, Cutting Fluids and Coolants; Cahners Books: Boston, MA, 1973.

(10) Benito, J. M.; Cambiella, A.; Lobo, A.; Gutiérrez, G.; Coca, J.; Pazos, C. Formulation, Characterization and Treatment of Metalworking Oil-in-Water Emulsions. Clean Technol. Environ. Policy. 2010, 12, 31.

(11) Zimmerman, J. B.; Clarens, A. F.; Hayes, K. F.; Skerlos, S. J. Design of Hard Water Stable Emulsifier Systems for Petroleum and Bio-Based Semi-Synthetic Metalworking Fluids. Environ. Sci. Technol. 2003, 37, 5278.

(12) Greeley, M.; Rajagopalan, N. Impact of Environmental Contaminants on Machining Properties of Metalworking Fluids. Tribol. Int. 2004, 37, 327.

(13) Zhang, D.; Lin, Y.; Li, A.; Tarasov, V. V. Emulsification for Castor Biomass Oil. Front. Chem. Sci. Eng. 2011, 5, 96. 
(14) Byers, J. P. Metal Working Fluids; 2nd ed.; Taylor \& Francis Group: Boca Raton, FL, 2006.

(15) Matthiessen, M. K.; Larney, F. J.; Selinger, L. B.; Olson, A. F.; Influence of Loss-onIgnition Temperature and Heating Time on Ash Content of Compost and Manure. Commun. Soil Sci. Plant Anal. 2005, 36, 2573.

(16) Griffin, W. C. Classification of Surface-Active Agents by 'HLB', J. Soc. Cosmet. Chem. 1949, 1,311 .

(17) ICI Americas Inc Home Page

http://www.firp.ula.ve/archivos/historicos/76_Book_HLB_ICI.pdf, (accessed Mar 2012).

(18) Assenhaimer, C.; Machado, L.; Glasse, B.; Fritsching, U.; Guardani, R. Use of a Spectroscopic Sensor to Monitor Droplet Size Distribution in Emulsions Using Neural Networks. Can. J. Chem. Eng. 2014, 92, 318.

(19) Glasse, B.; Assenhaimer, C.; Guardani, R.; Fritsching, U. Analysis of the Stability of Metal Working Fluid Emulsions by Turbidity Spectra. Chem. Eng. Technol. 2013, 36, 1.

(20) Zhmud, B. V.; Tiberg , F.; Kizling, J. Dynamic Surface Tension in Concentrated Solutions of $\mathrm{C}_{\mathrm{n}} \mathrm{E}_{\mathrm{m}}$ Surfactants: A Comparison between the Theory and Experiment. Langmuir. 2000, 16, 2557.

(21) Griffin, W. C. Calculation of HLB values of Non-Ionic Surfactants. J. Soc. Cosmet. Chem. $1954,5,249$.

(22) Deluhery, J.; Rajagopalan, N. A Turbidimetric Method for the Rapid Evaluation of MWF Emulsion Stability, Colloids Surf., A. 2005, 256, 145. 
(23) Binks B.P.; Fletcher P.D.I.; Taylor D.J.F. Temperature Insensitive Microemulsions. Langmuir. 1997, 13, 7030.

(24) Salager, J. L.; Antón, R. E.; Sabatini, D. A.; Harwell, J.H.; Acosta, J. E.; Tolosa, L. I. Enhancing Solubilization in Microemulsions-State of the Art and Current Trends. J. Surfact. Deterg. 2005, 8, 3 .

(25) Salager, J. L.; Marquez, N.; Graciaa, A.; Lachaise, L. Partitioning of Ethoxylated Octylphenol Surfactants in Microemulsion-Oil-Water Systems: Influence of Temperature and Relation Between Partitioning Coefficient and Physicochemical Formulation. Langmuir. 2000, $16,5534$.

(26) Totten George, E.; Westbrook Steven, R.; Shah Rajesh, J. Metalworking and Machining Fluids. Manuals 37: Fuels and Lubricants Handbook. ASTM International: Danvers, MA. 2003. 
Tables:

Table 1. Chemical analytical and surface tension data for SBO identified according to their source.

\begin{tabular}{|c|c|c|c|c|c|c|c|c|c|c|c|c|c|}
\hline SBO & \multicolumn{4}{|c|}{ Volatile Solids, w/w $\%^{\mathrm{a}}$} & \multicolumn{4}{|c|}{$\mathrm{C}, \mathrm{w} / \mathrm{w} \%^{\mathrm{a}}$} & \multicolumn{2}{|c|}{$\mathrm{N}, \mathrm{w} / \mathrm{w} \%^{\mathrm{a}}$} & \multicolumn{3}{|c|}{$\mathrm{C} / \mathrm{N}$} \\
\hline FORSUD & \multicolumn{4}{|c|}{84.6} & \multicolumn{4}{|c|}{$45.07 \pm 0.12$} & \multicolumn{2}{|c|}{$7.87 \pm 0.12$} & \multicolumn{3}{|c|}{5.73} \\
\hline CVDF & \multicolumn{4}{|c|}{72.7} & \multicolumn{4}{|c|}{$35.47 \pm 0.09$} & \multicolumn{2}{|c|}{$4.34 \pm 0.17$} & \multicolumn{3}{|c|}{8.17} \\
\hline $\mathrm{CV}$ & \multicolumn{4}{|c|}{72.1} & \multicolumn{4}{|c|}{$38.25 \pm 0.09$} & \multicolumn{2}{|c|}{$4.01 \pm 0.03$} & \multicolumn{3}{|c|}{9.54} \\
\hline Cvla & \multicolumn{4}{|c|}{99.1} & \multicolumn{4}{|c|}{$52.53 \pm 0.01$} & \multicolumn{2}{|c|}{$5.23 \pm 0.11$} & \multicolumn{3}{|c|}{10.04} \\
\hline & \multicolumn{13}{|c|}{ Functional groups $\mathrm{s}^{\mathrm{b}}$ concentration as mole fraction of total organic $\mathrm{C}$} \\
\hline & Af & $\mathrm{Nr}$ & $\mathrm{OMe}$ & OR & $\mathrm{OCO}$ & $\mathrm{Ph}$ & $\mathrm{PhOH}$ & $\mathrm{PhO}$ & $\mathrm{COOH}$ & $\mathrm{CON}$ & $\mathrm{C}=\mathrm{O}$ & $\mathrm{Af} / \mathrm{Ar}$ & LH \\
\hline FORSUD & 0.43 & 0.10 & 0.04 & 0.10 & 0.03 & 0.10 & 0.02 & 0.0 & 0.07 & 0.09 & 0.01 & 3.3 & 9.3 \\
\hline CVDF & 0.31 & 0.08 & 0.00 & 0.20 & 0.07 & 0.16 & 0.06 & 0.02 & 0.09 & 0.01 & 0.00 & 1.3 & 5.3 \\
\hline $\mathrm{CV}$ & 0.37 & 0.07 & 0.00 & 0.14 & 0.04 & 0.13 & 0.05 & 0.02 & 0.12 & 0.01 & 0.05 & 1.8 & 3.6 \\
\hline CVla & 0.28 & 0.07 & 0.00 & 0.14 & 0.06 & 0.22 & 0.06 & 0.0 & 0.11 & 0.00 & 0.01 & 0.8 & 4.5 \\
\hline
\end{tabular}

${ }^{\mathrm{a} C}$ Concentration values referred to dry matter: averages and standard deviation calculated over triplicates.

${ }^{\mathrm{b}} \mathrm{PhOY}=$ alkyl phenyl ether or dipheny ether; $\mathrm{Af} / \mathrm{Ar}=\mathrm{Af} /(\mathrm{Ph}+\mathrm{PhOH}+\mathrm{PhOY}) ; \mathrm{LH}=$ liphophilic to hydrophilic $\mathrm{C}$ ratio; liphophilic $\mathrm{C}=$ sum of aliphatic (Af), aromatic $(\mathrm{Ph})$, methoxy $(\mathrm{OMe})$, amide $(\mathrm{CON})$, amine $(\mathrm{NR})$, alkoxy (RO), phenoxy (PhOY) and anomeric (OCO) $\mathrm{C}$ atoms; hydrophilic $\mathrm{C}=$ sum of carboxylic acid $(\mathrm{COOH})$, phenol $(\mathrm{PhOH})$ and ketone $(\mathrm{C}=\mathrm{O}) \mathrm{C}$. 
Table 2. Data calculated from experimental surface tension measurements

\begin{tabular}{|c|c|c|c|c|c|c|}
\hline & $-\mathrm{d} \gamma_{1} / \mathrm{dCs}^{\mathrm{c}}$ & $\mathrm{R}_{1}{ }^{\mathrm{c}}$ & $\mathrm{Cs}_{1}{ }^{\mathrm{d}}$ & $\gamma_{\mathrm{Cs} 1}{ }^{\mathrm{e}}$ & $-\mathrm{d} \gamma_{2} / \mathrm{dCs}^{\mathrm{c}}$ & $\mathrm{R}_{2}{ }^{\mathrm{c}}$ \\
\hline FORSUD & $\begin{array}{c}11.5 \pm 0.9 \\
(0-1 ; 3)\end{array}$ & 0.995 & 1.0 & 48.9 & $\begin{array}{c}0.68 \pm 0.06 \\
(1-10 ; 8)\end{array}$ & 0.749 \\
\hline $\mathrm{CVDF}$ & $\begin{array}{c}5.3 \pm 0.7 \\
(0-3 ; 3)\end{array}$ & 0.999 & 3.1 & 61.8 & $\begin{array}{c}0.42 \pm 0.05 \\
(3-10 ; 8)\end{array}$ & 0.969 \\
\hline CVla & $\begin{array}{c}5.0 \pm 0.5 \\
(0-2 ; 4)\end{array}$ & 0.971 & 2.1 & 61.2 & $\begin{array}{c}0.94 \pm 0.12 \\
(2-9.76 ; 5)\end{array}$ & 0.938 \\
\hline
\end{tabular}

${ }^{\mathrm{c}}$ Slope values $\left(-\mathrm{d} \gamma_{1} / \mathrm{dCs}, \mathrm{mN} / \mathrm{L} / \mathrm{m}^{\prime} \mathrm{g}, \mathrm{i}=1,2\right)$ and standard deviations for the two linear tracts of each $\gamma$-Cs plot, associated Coefficient Determination $\left(R_{i}, i=1,2\right)$, and between parenthesis concentration range followed by number of experimental points in the regression analysis.

${ }^{\mathrm{d}}$ Concentration $(\mathrm{g} / \mathrm{L})$ at which slope change occurs.

${ }^{\mathrm{e}} \mathrm{Surface}$ tension value at slope change point.

Table 3. Number of days of stability for emulsions containing the four SBO at different $\mathrm{R}$ values and total surfactant concentration; ST standing for over 15 days stability.

\begin{tabular}{|c|c|c|c|c|c|c|c|c|c|c|c|c|c|c|c|c|c|c|c|c|c|c|c|}
\hline \multirow{2}{*}{$100 \mathrm{R}$} & & \multicolumn{4}{|c|}{ CVla } & \multicolumn{6}{|l|}{$\mathrm{CV}$} & \multicolumn{5}{|c|}{ CVDF } & \multicolumn{7}{|c|}{ FORSUD } \\
\hline & & 61 & 57 & 51 & 47 & 85 & 72 & 61 & 57 & 51 & 47 & 72 & 61 & 57 & 51 & 47 & 100 & 85 & 72 & 67 & 61 & 59 & 51 \\
\hline \multicolumn{2}{|l|}{ HLB $^{\mathbf{a}}$} & 18 & 19 & 20 & 21 & 16 & 17 & 18 & 19 & 20 & 21 & 17 & 18 & 19 & 20 & 21 & 15 & 16 & 17 & 17.5 & 18 & 18.5 & 19 \\
\hline \multirow{4}{*}{ 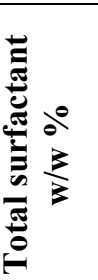 } & 2 & - & 0 & 0 & 0 & - & - & - & - & - & - & - & - & - & - & - & - & - & - & 2 & - & - & - \\
\hline & 3 & - & 5 & ST & 4 & - & 1 & 5 & 1 & 1 & 1 & 1 & ST & ST & ST & 1 & - & - & 0 & 3 & 1 & - & - \\
\hline & 5 & 6 & ST & ST & ST & 7 & ST & ST & ST & ST & ST & ST & ST & $\mathrm{ST}$ & ST & ST & 1 & 2 & ST & $\mathrm{ST}$ & ST & 6 & 6 \\
\hline & 8 & ST & ST & ST & ST & ST & ST & ST & ST & ST & ST & 2 & ST & $\mathrm{ST}$ & ST & ST & ST & ST & ST & ST & ST & ST & ST \\
\hline
\end{tabular}

${ }^{\mathrm{a} C}$ Calculated from Eq. 2. 


\section{List of Figure Captions:}

Figure 1. Virtual molecular fragments for $\mathrm{SBO}$ isolated from municipal biowastes fitting analytical data for $\mathrm{C}$ types and functional groups. $\mathrm{H}$ bonded to $\mathrm{C}$ omitted; sinusoidal bold lines indicate other fragments $\mathrm{C}, \mathrm{O}, \mathrm{N}$ atoms.

Figure 2. Surface tension $(\gamma)$ vs. added SBO concentration (Cs).

Figure 3. Optical microscope images of emulsions containing CV at 5 (a) and 3 (b) w/w \% total surfactant concentration and $\mathrm{R}=0.618$ days after preparation.

Figure 4. Particles size distribution and Sauter mean diameter (SMD) for 8 days aged emulsions containing $3 \%$ and $5 \%$ total surfactant concentration and the four SBO at $\mathrm{R}$ values of 0.57 for CVDF, 0.51 for CVla, 0.61 for CV, and 0.67 for FORSUD.

Figure 5. Emulsion stability after $4000 \mathrm{rpm}$ centrifugation for freshly prepared mixtures; mean value and standard deviation for separated volume fraction $(\mathrm{F})$ of the total mixture volume versus total surfactant concentration for emulsions containing $\operatorname{SDS}$ at $\mathrm{R}=0.14$, and for emulsions prepared with SBO at R values of 0.61 for $\mathrm{CV}, 0.51$ for CVla, 0.57 for CVDF, and 0.67 for FORSUD. Columns with no letter in common indicate significantly different values at $95 \%$ confidence level; i.e. $\mathrm{a}>\mathrm{b}>\mathrm{c}$.

Figure 6. Shear stress versus shear rate for emulsions containing $5 \% \mathrm{w} / \mathrm{w}$ total surfactant concentration and $\mathrm{SBO}$ at $\mathrm{R}$ values of 0.61 for $\mathrm{CV}, 0.51$ for $\mathrm{CVla}, 0.57$ for $\mathrm{CVDF}, 0.67$ for FORSUD. 
Figure 7. Viscosity over time at speed of $20 \mathrm{rpm}$ for emulsions containing the SBO at $5 \%$ total surfactant concentration and $\mathrm{R}$ values of 0.61 for $\mathrm{CV}, 0.51$ for CVla, 0.57 for CVDF, 0.67 for FORSUD, and 0.14 for SDS. 